\title{
Complex Generalised Fuzzy Soft Sets and its Application
}

\author{
ABD ULZEEZ M. J. S. ALKOURI \\ Mathematics Department, Science College \\ Ajloun National University \\ P.O. Box 43, 26810, Ajloun \\ JORDAN
}

\begin{abstract}
Human knowledge and mentality of experts may be changed with the time making the time a very important factor to the decision-makers. Therefore, different decisions for exact problem can be made by decision-makers in different times. We introduce here a new mathematical tool called complex generalized fuzzy soft set (CGFSS), which is a combination of the concept of generalized fuzzy soft set (GFSS) and complex fuzzy set (CFS). The importance of CGFSS may be appeared in the ability to convey the parametric nature in the concept of GFSS that happening periodically without losing the full meaning of human knowledge. While the uncertainty values lie in GFSS may be affected by different factors/phases/levels, CGFSS represents two values for each parameter (i) the degree of membership "belongingness of uncertainty and periodicity for elements in universe of discourse" and (ii) the degree of uncertainty and periodicity for the possibility of such belongingness which are represented by using complex membership form. Some CGFSS's basic operations and its properties are introduced with the definition of relation on this tool and its application to illustrate the novelty of CGFSS in the decision-making problem. Finally, a comparison between several uncertainty sets and CGFSS is illustrated.
\end{abstract}

Keywords: Fuzzy soft set, complex fuzzy soft set, generalized fuzzy soft set, complex generalized fuzzy soft set, and relation.

Received: April 17, 2019. Revised: May 4, 2020. Accepted: June 1, 2020. Published: June 8, 2020.

\section{Introduction}

Ramot et al. (2002) generalized the theory of complex fuzzy sets for dealing with uncertainties and periodicity information simultaneously. Many researchers have been used successfully Ramot's idea [1,2,3,10-23], which is extending the interval $[0,1]$ to of the membership function's range, in several areas like; decision-making problems, forecasting, medical, multiple periodic factor prediction problems, and PSO-RLSE to solve some prediction, time series forecasting, adaptive image noise-canceling, and function approximation problems. In the reference [24], Ramot and other researchers introduced the concept of complex fuzzy relation (CFR), where the value of absence or presence of association, interconnectedness, or interaction and the phase of association, interconnectedness or interaction, among two or more sets can be conveyed by using the concept of CFRs". Recently, some researchers have been successfully generalized and applied the concept of CFR. To name but a few: Alkouri and Salleh [10] introduced complex Atanasov intuitionistic fuzzy relation and applied it in multi attributes decisionmaking (MADM) problems. In 2018, Al-Qudah and Hassan [25] presented complex multi-fuzzy relation (CMFR) for Decision Making, where the innovation of CMFR can be seen in the capability of complex multi-membership functions to attain an extra range of values for uncertainty information indexed by periodicity in nature.

Maji et al [4-6] studied the theory of soft sets, initiated by Molodtsov [7] and applied this theory to deal with some decision-making problems. The notion of the fuzzy soft set had been also presented by Maji et al., which is a mixture of a fuzzy set and soft set. In 2009, Kong et al. [8] have used the fuzzy soft set to deal with problems in the decision-making field. Furthermore, Majumdar and Samanta [9] introduced a generalized fuzzy soft set. The fuzzy soft set gets an additional degree. This degree is committed to the parametrization and represents the degree of possibility of belongingness of elements of fuzzy sets. Also in [9] Relations and their properties, similarity and an application in medical diagnosis and decision making problems on generalized fuzzy soft sets are studied.

Here, we have extended the notion of generalized fuzzy soft sets [9] to the complex realm by adding the periodicity semantics in both degrees of belongingness and degree of possibility of belongingness. We incorporate two concepts, complex fuzzy set, and generalized fuzzy soft set. We attached the degree of representing the possibility of belongingness of elements of complex fuzzy sets. We depend on the expert's opinion to solve a problem in decision making. Expert's opinion are changeable with an object affected by 
some factors (e.g. time). Each object and factor are represented by GFSS (parametric in nature) depends on the expert's opinion. The object in CGFS will be represented by amplitude terms and the factor will be represented by using the phase terms. Both object and factor will convey belongingness and possibility of belongingness values for each element in universe $\mathrm{W}$ of the expert's opinion. To show how we characterize this type of information, this research presents the properties of the CGFSS. The phase term conveys the values of factor may affecting the amplitude term. Since this factor is represented by GFSS, these values must have belongingness and possibility of belongingness values (See Application 4.1.). Therefore, CGFSS is a more representative and wider range. It includes periodicity and uncertainty in the choice of a complex fuzzy set matching to each value of the parameter. A decision is given to select a proper machine by using the CGFS tool by generalizing the presented model in [9] to the realm of complex numbers.

We organize this manuscript as follows: In Section 2, some results and definitions are recalled which is related to the current results. In Section3, a definition of a complex generalized fuzzy soft set is formalized and studied some of its properties. In Section 4, an application of the complex generalized fuzzy soft set is introduced by using a new proper model in the decision-making problem. In section 5, we are presenting a comparative study among different uncertainty sets (fuzzy set, fuzzy soft set, generalized fuzzy soft set, and complex fuzzy set) with CGFSS. (See section 6 for the summary).

\section{Preliminaries}

The current part of this research present and recollect some basic operations and relevant definitions.

Definition 2.1 [26] A fuzzy set $A$ in a universe of discourse $U$ is characterized by a membership function $\mu_{A}(x)$ that takes values in the interval [0, 1].

Definition 2.2 [7] Let $U$ be an initial set and $E$ be a set of parameters. Let $P(U)$ denote the power set of $U$, and let $A \subset E$. A pair $(F, A)$ is called a soft set over $U$, where $F$ is a mapping given by $F: A \rightarrow P(U)$

Definition 2.3 [4] Let $U$ an initial set and $E$ be a set of parameters. Let $F(U)$ denote the fuzzy power set of $U$, and let $A \subset E$. A pair ( $F, A$ ) is called a fuzzy soft set over $U$, where $F$ is a mapping given by $F: A \rightarrow F(U)$.
Definition 2.4 [24] A complex fuzzy set A, defined on a universe of discourse $U$, is characterized by a membership function $\mu_{A}(x)$, that assigns to any element $x \in U$ a complex-valued grade of membership in A. By definition, the values of $\mu_{A}(x)$ , may receive all lying within the unit circle in the complex plane, and are thus of the form $\mu_{A}(x)=r_{A}(x) e^{i \theta_{A}(x)}$, where $i=\sqrt{-1}$, each of $r_{A}(x)$ and $\theta_{A}(x)$ are both real-valued, and $r_{A}(x) \in[0,1]$. The CFS $A$ may be represented as the set of ordered pairs $A=\left\{\left(x, \mu_{A}(x)\right): x \in U\right\}$.

Definition 2.5 [24] A complex fuzzy complement of $A$ may be represented as follows:

$$
\bar{A}=\left\{\left(x, \mu_{\bar{A}}(x)\right): x \in U\right\}=\left\{\left(x, r_{\bar{A}}(x) \cdot e^{i \bar{A}_{\bar{A}}(x)}\right): x \in U\right\},
$$

where

$$
r_{\bar{A}}(x)=1-r_{A}(x)
$$

and

$\theta_{\bar{A}}(x)=\omega_{A}(x)$, or $=2 \pi-\theta_{A}(x)$, or $=\pi+\theta_{A}(x)$.

Definition 2.6. [24] Let $A$ and $B$ be two complex fuzzy sets on $X, \mu_{A}(x)=r_{A}(x) e^{i \theta_{A}(x)}$ and $\mu_{B}(x)=r_{B}(x) e^{i \theta_{B}(x)}$ their membership functions, respectively. We say that $A$ is greater than $B$, denoted by $A \supseteq B$ or $B \subseteq A$, if for any $x \in X$, $r_{A}(x) \leq r_{B}(x)$, and $\theta_{A}(x) \leq \theta_{B}(x)$.

Definition 2.7 [24] Let $A$ and $B$ be two complex fuzzy sets on $X$, with complex-valued membership function $\mu_{A}(x)$ and $\mu_{B}(x)$. The complex fuzzy union of $A$ and $B$ denoted $A \cup B$, is specified by a function

$u:\{a|a \in C| a \mid, \leq 1\} *\{b|\boldsymbol{b} \in \mathcal{C}| b \mid, \leq 1\} \rightarrow\{d \mid d \in$ $C,|d| \leq 1\}$.

$u$ assigns a complex value, $\mathrm{u}\left(\mu_{A}(x), \mu_{B}(x)\right)=$ $\mu_{A \cup B}(x)$ to all $x$ in $X$.

The complex fuzzy union function u must satisfy at least the following axiomatic requirements, for any $a, b, c, d \in\{x|x \in C| x \mid, \leq 1\}$

Axiom 1 (boundary conditions), $u(a, 0)=a$.

Axiom 2 (monotonicity): $|b| \leq|d|$ implies $|u(a, b)| \leq|u(a, d)|$.

Axiom 3 (commutativity): $u(a, b)=u(b, a)$.

Axiom 4 (associativity): $u(a, u(b, d))=u(u(a, b), d)$.

Axiom 5 (continuity): $u$ is a continuous function.

Axiom 6 (superidempotency): $|\mathrm{u}(\mathrm{a}, \mathrm{a})|>|a|$.

Axiom 7 (strict monotonicity): $|a| \leq|c|$ and $|b| \leq$ $|d| \Rightarrow|u(a, b)| \leq|u(c, d)|$.

Definition 2.8 [24] let $A$ and $B$ be two complex fuzzy sets on $X$, with complex-valued membership function $\mu_{A}(x)$ and $\mu_{B}(x)$. The complex fuzzy 
intersection of $A$ and $B$ denoted $A \cap B$, is specified by a function

$i:\{a|a \in C,| a \mid \leq 1\}^{*}\{b|b \in C| b \mid, \leq 1\} \rightarrow\{d \mid d \in C$ $,|d| \leq 1\}$.

$i$ assigns a complex value, $\mathrm{u}\left(\mu_{A}(x), \mu_{B}(x)\right)=$ $\mu_{A \cap B}(x)$ to all $x$ in $X$.

The complex fuzzy intersection function $i$ must satisfy at least the following axiomatic requirements, for any $a, b, c, d \in\{x|x \in C| x \mid, \leq$ $1\}$

Axiom 1 (boundary conditions): $|b|=1$, if $|i(a, b)|=|a|$.

Axiom 2 (monotonicity): $|b| \leq|d|$ implies $|i(a, b)| \leq|i(a, d)|$.

Axiom 3 (commutativity): $\mathrm{i}(\mathrm{a}, \mathrm{b})=\mathrm{i}(b, a)$.

Axiom 4 (associativity): $i(a, i(b, d))=i(i(a, b), d)$.

Axiom 5 (continuity): $i$ is a continuous function.

Axiom 6 (superidempotency): $|i(a, a)|<|a|$.

Axiom 7 (strict monotonicity): $|a| \leq|c|$ and $|b| \leq$ $|d| \Rightarrow|i(a, b)| \leq|i(c, d)|$.

Table 1 shows some examples of $s$-norms and $t$ norms under CFS.

Table 1. Some Examples of $s$-norms and $t$-norm

\begin{tabular}{|c|c|}
\hline 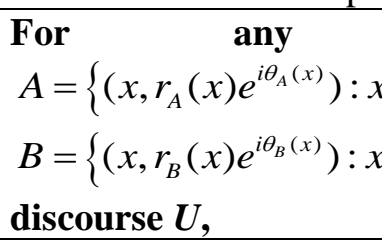 & $\begin{array}{l}\text { two } \\
\{\in X\} \text { and } \\
\{\in X\} \text { in a }\end{array}$ \\
\hline $\begin{array}{l}\text { Basic s-norm: } \\
A \cup B= \\
\left\{\left\langle x, \max \left(r_{A}(x), r_{B}(x)\right),\right.\right. \\
\left.\left.\max \left(\theta_{A}(x), \theta_{B}(x)\right)\right\rangle: x \in X\right\}\end{array}$ & $\begin{array}{l}\text { Basic } t \text {-norm: } \\
A \cap B= \\
\left\{\left\langle x, \min \left(r_{A}(x), r_{B}(x)\right),\right.\right. \\
\left.\left.\min \left(\theta_{A}(x), \theta_{B}(x)\right)\right\rangle: x \in X\right\}\end{array}$ \\
\hline $\begin{array}{l}\text { Yager } S \text {-norm: } \\
s\left(\left(a e^{a^{\prime}}\right),\left(b e^{b^{\prime}}\right)\right)= \\
s\left(s^{r}{ }_{\sigma}(a, b), s^{\theta}{ }_{\sigma}\left(a^{\prime}, b^{\prime}\right)\right) \text {, } \\
\text { where } \\
s^{r}{ }_{\sigma}(a, b)= \\
\min \left(1,\left(a^{\sigma}+b^{\sigma}\right)^{1 / \sigma}\right) \\
\text { and } \\
s^{\theta}{ }_{\sigma}\left(a^{\prime}, b^{\prime}\right)= \\
\min \left(1,\left(a^{\prime}{ }^{\sigma}+b^{\prime}\right)^{1 / \sigma}\right) \\
\text { with } \varpi \in(0, \infty) .\end{array}$ & $\begin{array}{l}\text { Yager T-norm: } \\
t\left(\left(a e^{a^{\prime}}\right),\left(b e^{b^{\prime}}\right)\right)= \\
t\left(t^{r}(a, b), t^{\theta}{ }_{\sigma}\left(a^{\prime}, b^{\prime}\right)\right), \\
\text { where } \\
{ }_{t^{r}}^{r}(a, b)= \\
1-\min \left(1,\left((1-a)^{\sigma}+(1-b)^{\sigma}\right)^{1 / \pi}\right) \\
\text { and } \\
{ }_{t^{\theta}}{ }_{\sigma}\left(a^{\prime}, b^{\prime}\right)= \\
\min \left(1,\left(\left(a^{\prime}\right)^{\sigma}+\left(b^{\prime}\right)^{\sigma}\right)^{1 / \sigma}\right. \\
\text { with } \varpi \in(0, \infty) .\end{array}$ \\
\hline
\end{tabular}

Definition 2.9 [9]. Let $U=\left\{x_{1}, x_{2}, \ldots, x_{n}\right\}$ be the universal set of elements and $E=\left\{e_{1} ; e_{2} ; \ldots ; e_{m}\right\}$ be the universal set of parameters. The pair $(U, E)$ will be called a soft universe. Let $F: E \rightarrow I^{U}$ and $\mu$ be a fuzzy subset of $E$, i.e $\mu: E \rightarrow I=[0,1]$, where $I^{U}$ is the collection of all fuzzy subsets of $U$. Let $F_{\mu}$ be the mapping $F_{\mu}: E \rightarrow I^{U} \times I$ be a function defined as follows:

$F_{\mu}(e)=(F(e), \mu(e))$ where $F(e) \in I^{U}$ : Then $F_{\mu}$ is called a generalized fuzzy soft set (GFSS) over the soft universe $(U, E)$. Here for each parameter $\quad e_{j}, \quad F_{\mu}\left(e_{j}\right)=\left(F\left(e_{j}\right), \mu\left(e_{j}\right)\right)$ indicates not only the degree of membership $e$ belongingness the elements of $U$ in $F\left(e_{j}\right)$ but also the degree of possibility of such belongingness of uncertainty which is represented by $\mu\left(e_{j}\right)$.

Example 2.1. Let $U=\left\{x_{1}, x_{2}, x_{3}\right\}$ be a set of three shirts. Let $E=\left\{e_{1} ; e_{2} ; e_{3}\right\}$ be a set of qualities where $\mathrm{e}_{1}=$ bright; $\mathrm{e}_{2}=$ cheap; $\mathrm{e}_{3}=$ colorful. Let $\mu: E \rightarrow I$ be defined as follows: $\mu\left(e_{1}\right)=0.2$; $\mu\left(e_{2}\right)=0.5 ; \mu\left(e_{3}\right)=0.8$.

We define a function $F_{\mu}: E \rightarrow I^{U} \times I$ be defined as follows:

$$
\begin{gathered}
F_{\mu}\left(e_{1}\right)=\left(\left\{\frac{x_{1}}{0.2}, \frac{x_{2}}{0.5}, \frac{x_{3}}{0.1}\right\}, 0.2\right), \\
F_{\mu}\left(e_{2}\right)=\left(\left\{\frac{x_{1}}{0}, \frac{x_{2}}{0.2}, \frac{x_{3}}{0.9}\right\}, 0.5\right), \text { and } \\
F_{\mu}\left(e_{3}\right)=\left(\left\{\frac{x_{1}}{0.9}, \frac{x_{2}}{0.6}, \frac{x_{3}}{0.2}\right\}, 0.8\right) .
\end{gathered}
$$

Then the family $\left\{F_{\mu}\left(e_{j}\right): j=1,2,3\right.$. $\}$ of $I^{U} \times I$ is a generalized fuzzy soft set.

Definition 2.10. [9]. Let $F_{\mu}(e)$ and $G_{\delta}(e)$ be two GFSS over $(U, E)$. Now $F_{\mu}(e)$ is said to be a generalized fuzzy soft subset of $G_{\delta}(e)$ if

(i) $\mu$ is a fuzzy subset of $\delta$.

(ii) $F(e)$ is also a fuzzy subset of $G(e), \forall e \in E$. In this case we write $F_{\mu}(e) \subseteq G_{\delta}(e)$.

Note. Let $\bigcirc$ and $*$ be two binary operations on $[0,1]$ defined as $s$-norm and $t$-norm, respectively.

Definition 2.11. [9]. Union of two GFSS $F_{\mu}(e)$ and $G_{\delta}(e)$, denoted by $F_{\mu}(e) \tilde{U} G_{\delta}(e)$

$$
\begin{array}{lr}
\text { is a GFSS } H_{v}(e), & \text { defined as } \\
H_{v}(e): E \rightarrow I^{U} \times I \text { such that } & \\
H_{v}(e)=(H(e), v(e)) & \text { where } \\
H(e)=F(e) \circ G(e) & \text { and } \\
v(e)=\mu(e) \circ \delta(e) . &
\end{array}
$$


Definition 2.12. [9]. The intersection of two GFSS $F_{\mu}(e)$ and $G_{\delta}(e)$, denoted by $F_{\mu}(e) \tilde{\cap} G_{\delta}(e)$ is a GFSS $H_{v}(e)$, defined as $H_{v}(e): E \rightarrow I^{U} \times I$ such that

$$
\begin{aligned}
& H_{v}(e)=(H(e), v(e)) \\
& H(e)=F(e) * G(e) \text { and } \\
& v(e)=\mu(e) * \delta(e) .
\end{aligned}
$$$$
\text { where }
$$

In this paper, we use the Normalized Euclidean distance between two complex fuzzy sets [24].

For any two complex fuzzy sets $A=\left\{\left(x, r_{A}(x) e^{i \theta_{A}(x)}\right): \forall x \in U\right\}$ and $B=\left\{\left(x, r_{B}(x) e^{i \theta_{B}(x)}\right): \forall x \in U\right\}$, then the Normalized Euclidean distance is given by $q_{C F}(A, B)=\sqrt{\frac{1}{2 n} \sum_{i=1}^{n}\left[\left(r_{A}\left(x_{i}\right)-r_{B}\left(x_{i}\right)\right)^{2}+\frac{1}{2 \pi^{2}}\left(\theta_{A}\left(x_{i}\right)-\theta_{B}\left(x_{i}\right)\right)^{2}\right]}$

\section{Complex Generalized Fuzzy Soft Sets}

As a result, the concept of CGFSS is introduced and its basic theoretical operations are defined and studied in this section. Some numerical examples and theorems are illustrated in the realm of CGFSS. Definition 3.1. Let $W=\left\{x_{1}, x_{2}, \ldots, x_{n}\right\}$ be the universal set, and $E=\left\{e_{1}, e_{2}, \ldots ; e_{m}\right\}$ be the set of parameters. The pair $(W, E)$ is called a soft universe. Let $C F_{\mu}(e): E \rightarrow I_{c}^{W}$ and $\mu$ be a complex fuzzy subset of $E$. i.e $\mu: E \rightarrow I_{c}=\{a:|a| \leq 1, \forall a \in \mathbb{C}\}=r e^{i \theta}$, where both $r$ and $\theta$ values in $[0,1]$, and $\boldsymbol{I}_{c}^{W}$ is the collection of all complex fuzzy subsets of $W$. Let the mapping $C F_{\mu}(e): E \rightarrow I_{c}^{W} \times I_{c}$ be a function defined as follows:

$C F_{\mu}(e)=(C F(e), \mu(e))$ where $C F(e) \in I_{c}^{W}$ : Then $C F_{\mu}(e)$ is named a complex generalized fuzzy soft set (CGFSS) over the soft universe $(W, E)$.

For each parameter $e_{j}, C F_{\mu}\left(e_{j}\right)=\left(C F\left(e_{j}\right), \mu\left(e_{j}\right)\right)$ point to not only the degree of membership $e$ belongingness of $j$ uncertainty and periodicity the elements of $W$ in $C F\left(e_{j}\right)$ but also the degree of possibility of such belongingness of uncertainty and periodicity which is represented by $\mu\left(e_{j}\right)$.

Example 3.2. Let $W=\left\{x_{1}, x_{2}, x_{3}\right\}$ be a set of three shirts. Let $E=\left\{e_{1}, e_{2}, e_{3}\right\}$ be a set of features, where $\mathrm{e}_{1}=$ dazzling; $\mathrm{e}_{2}=$ colorful; $\mathrm{e}_{3}=$ expensive. Let $\mu: E \rightarrow I_{c}=\{a:|a| \leq 1, \forall a \in \mathbb{C}\}$ be getting the following values:

$\mu\left(e_{1}\right)=0.2 e^{i 2 \pi(0.3)} ; \mu\left(e_{2}\right)=0.5 e^{i 2 \pi(0.3)} ; \mu\left(e_{3}\right)=0.7$ $e^{i 2 \pi(0.3)}$. So, we may be giving a function $C F_{\mu}(e): E \rightarrow I_{c}^{W} \times I_{c}$ as follows:

$$
\begin{aligned}
& C F_{\mu}\left(e_{1}\right)=\left(\left\{\frac{x_{1}}{0.7 e^{i 2 \pi(0.2)}}, \frac{x_{2}}{0.4 e^{i 2 \pi(0.1)}}, \frac{x_{3}}{0.3 e^{i 2 \pi(0.1)}}\right\}, 0.2 e^{i 2 \pi(0.3)}\right) \\
& C_{\mu}\left(e_{2}\right)=\left(\left\{\frac{x_{1}}{0.1 e^{i 2 \pi(0.2)}}, \frac{x_{2}}{0.2 e^{i 2 \pi(0.1)}}, \frac{x_{3}}{0.9 e^{i 2 \pi(0.1)}}\right\}, 0.5 e^{i 2 \pi(0.3)}\right)
\end{aligned}
$$

$C F_{\mu}\left(e_{3}\right)=\left(\left\{\frac{x_{1}}{0.8 e^{i 2 \pi(0.2)}}, \frac{x_{2}}{0.5 e^{i 2 \pi(0.1)}}, \frac{x_{3}}{0.2 e^{i 2 \pi(0.1)}}\right\}, 0.7 e^{i 2 \pi(0.3)}\right)$

Then $C F_{\mu}\left(e_{j}\right)=\left(C F\left(e_{j}\right), \mu\left(e_{j}\right)\right)$ is a CGFSS over $(W, E)$.

Or we may represent it as a matrix form as follows: $C F_{\mu}\left(e_{j}\right)=\left(C F\left(e_{j}\right), \mu\left(e_{j}\right)\right)=$

$$
=\left(\begin{array}{llll}
0.7 e^{i 2 \pi(0.2)} & 0.4 e^{i 2 \pi(0.1)} & 0.3 e^{i 2 \pi(0.1)} & 0.2 e^{i 2 \pi(0.3)} \\
0.1 e^{i 2 \pi(0.2)} & 0.2 e^{i 2 \pi(0.1)} & 0.9 e^{i 2 \pi(0.1)} & 0.5 e^{i 2 \pi(0.3)} \\
0.8 e^{i 2 \pi(0.2)} & 0.5 e^{i 2 \pi(0.1)} & 0.2 e^{i 2 \pi(0.1)} & 0.7 e^{i 2 \pi(0.3)}
\end{array}\right),
$$

where the $j^{\text {th }}$ row vector represents $C F_{\mu}\left(e_{j}\right)$, the $j^{\text {th }}$ column vector represents $\boldsymbol{x}_{j}$, the last column represents the degree of $\mu$ and it is called complex membership matrix of $C F_{\mu}$.

Definition 3.3. Let $C F_{\mu}(e)$ and $C G_{\delta}(e)$ be two CGFSS over $(W, E)$. Now $C F_{\mu}(e)$ is supposed to be a complex generalized of the complex fuzzy soft subset of $C G_{\delta}(e)$ if

(i) $\mu$ is a complex fuzzy subset of $\delta$.

(ii) $C F(e)$ is also a complex fuzzy subset of $C G(e), \forall e \in E$. denoted by $C F_{\mu}(e) \subseteq C G_{\delta}(e)$.

Example 3.4. As in Example 3.2, let $C F_{\mu}(e)$ be the CGFSS over $(W, E)$ given in Example 3.2. Let $C G_{\delta}(e)$ be one more CGFSS over $(W, E)$ has the following values:

$$
\begin{aligned}
& C G_{\delta}\left(e_{1}\right)=\left(\left\{\frac{x_{1}}{0.2 e^{i 2 \pi(0.2)}}, \frac{x_{2}}{0.3 e^{i 2 \pi(0.1)}}, \frac{x_{3}}{0.1 e^{i 2 \pi(0.1)}}\right\}, 0.2 e^{i 2 \pi(0.3)}\right) \\
& C_{\delta}\left(e_{2}\right)=\left(\left\{\frac{x_{1}}{0.0 e^{i 2 \pi(0.2)}}, \frac{x_{2}}{0.1 e^{i 2 \pi(0.1)}}, \frac{x_{3}}{0.7 e^{i 2 \pi(0.1)}}\right\}, 0.4 e^{i 2 \pi(0.3)}\right)
\end{aligned}
$$


$C G_{\delta}\left(e_{3}\right)=\left(\left\{\frac{x_{1}}{0.7 e^{i 2 \pi(0.2)}}, \frac{x_{2}}{0.3 e^{i 2 \pi(0.1)}}, \frac{x_{3}}{0.1 e^{i 2 \pi(0.1)}}\right\}, 0.6 e^{i 2 \pi(0.3)}\right)$

. where $\delta \in I_{c}^{W}$ be defined as above:

Then $C G_{\delta}(e) \subseteq C F_{\mu}(e)$.

Note 3.5 ([11]). Let $c$ be a complex fuzzy complement and $\boldsymbol{g}$ be an increasing generator of $\boldsymbol{c}$. Let the binary operations $\bigcirc$ and $*$ be defined as: * is a t-norm and $\circ$ is an s-norm. Moreover (* ; $\circ ; \boldsymbol{c}$ ) develops a dual triple. Hereafter, we will consider such a dual triple as the general case.

Definition 3.6. The complement of $C_{\mu}(e)$ over $(W, E)$, denoted by $C F_{\mu}{ }^{c}(e)$, is defined by $C F_{\mu}{ }^{c}(e)=C G_{\delta}(e)$, where $\delta(e)=\mu^{c}(e)$ and $C G(e)=C F^{c}(e), \forall e \in E$.

Note 3.7. The equality $\left(C F_{\mu}^{c}\right)^{c}=C F_{\mu}$ holds.

Definition 3.8. Let $C F_{\mu}(e)$ and $C G_{\delta}(e)$ be two CGFSS over $(W, E)$, Then the union of $C F_{\mu}(e)$ and $C G_{\delta}(e)$, denoted by $C F_{\mu}(e) \tilde{U} C G_{\delta}(e)$, is a CGFSS $\mathrm{CH}_{V}(e)$ defined as $\mathrm{CH}_{v}(e): E \rightarrow I_{c}^{W} \times I_{c} \quad$ such that $\mathrm{CH}_{v}(e)=(\mathrm{CH}(e), v(e))$ where $C H(e)=C F(e) \circ C G(e)$ and $v(e)=\mu(e) \circ \delta(e)$.

Definition 3.9. Let $C F_{\mu}(e)$ and $C G_{\delta}(e)$ be two CGFSS over $(W, E)$. Then the intersection of two CGFSS $C F_{\mu}(e)$ and $C G_{\delta}(e)$, denoted by $C F_{\mu} \tilde{\cap} C G_{\delta}$ is a CGFSS $C H_{v}(e)$, defined as $\mathrm{CH}_{v}(e): E \rightarrow I_{c}^{W} \times I_{c}$ such that $\mathrm{CH}_{v}(e)=(C H(e), v(e))$ where $C H(e)=C F(e) * C G(e)$ and $v(e)=\mu(e) * \delta(e)$.

Example 3.10. Consider $C F_{\mu}(e)$ and $C G_{\delta}(e)$ as defined in Examples 3.4. Let the operation * defined on $\{a:|a| \leq 1, \forall a \in \mathbb{C}\}$ as follows: $a * b=$ (ab) $e^{i 2 \pi\left(\theta_{a} \theta_{b}\right)}$ and the operation $\circ$ defined on $\{a:|a| \leq 1, \forall a \in \mathbb{C}\}$ as follows: $a \circ b=(a+b-a b)$ $e^{i 2 \pi\left(\theta_{a}+\theta_{b}-\theta_{a} \theta_{b}\right)}$. Also, take $\boldsymbol{c}$ as $\left(a^{c}=(1-a) e^{i 2 \pi\left(1-\theta_{a}\right)}\right.$ ) Then $(* ; \circ ; c)$ forms a dual triple. Then,

$$
\begin{aligned}
& C F_{\mu}(e) \tilde{\cup} C G_{\delta}(e)= \\
& \left(\begin{array}{llll}
0.76 e^{i 2 \pi(0.36)} & 0.58 e^{i 2 \pi(0.19)} & 0.37 e^{i 2 \pi(0.19)} & 0.36 e^{i 2 \pi(0.51)} \\
0.1 e^{i 2 \pi(0.36)} & 0.28 e^{i 2 \pi(0.19)} & 0.97 e^{i 2 \pi(0.19)} & 0.64 e^{i 2 \pi(0.51)} \\
0.94 e^{i 2 \pi(0.36)} & 0.65 e^{i 2 \pi(0.19)} & 0.28 e^{i 2 \pi(0.19)} & 0.84 e^{i 2 \pi(0.51)}
\end{array}\right)
\end{aligned}
$$

$$
\begin{aligned}
& C F_{\mu}(e) \tilde{\cap} C G_{\delta}(e)= \\
& \left(\begin{array}{llll}
0.14 e^{i 2 \pi(0.04)} & 0.12 e^{i 2 \pi(0.01)} & 0.03 e^{i 2 \pi(0.01)} & 0.04 e^{i 2 \pi(0.09)} \\
0.0 e^{i 2 \pi(0.04)} & 0.02 e^{i 2 \pi(0.01)} & 0.63 e^{i 2 \pi(0.01)} & 0.16 e^{i 2 \pi(0.09)} \\
0.56 e^{i 2 \pi(0.04)} & 0.15 e^{i 2 \pi(0.01)} & 0.02 e^{i 2 \pi(0.01)} & 0.36 e^{i 2 \pi(0.09)}
\end{array}\right) \\
& C G_{\delta}^{c}(e)= \\
& \left(\begin{array}{llll}
0.8 e^{i 2 \pi(0.8)} & 0.7 e^{i 2 \pi(0.9)} & 0.9 e^{i 2 \pi(0.9)} & 0.8 e^{i 2 \pi(0.7)} \\
1.0 e^{i 2 \pi(0.8)} & 0.9 e^{i 2 \pi(0.9)} & 0.3 e^{i 2 \pi(0.9)} & 0.6 e^{i 2 \pi(0.7)} \\
0.3 e^{i 2 \pi(0.8)} & 0.7 e^{i 2 \pi(0.9)} & 0.9 e^{i 2 \pi(0.9)} & 0.4 e^{i 2 \pi(0.7)}
\end{array}\right)
\end{aligned}
$$

Definition 3.11. A CGFSS is said to be a complex generalized null fuzzy soft set, denoted by $C \phi_{\theta}(e)$ if $C \phi_{\theta}(e): E \rightarrow I_{c}^{W} \times I_{c}$ such that $C \phi_{\theta}(e)=(C F(e), \theta(e))$, where $C F(e)=\overline{0} \cdot e^{i 0(2 \pi)} \forall e \in E$ and $\theta(e)=0 \cdot e^{i 0(2 \pi)} \forall e \in E$.

Definition 3.12. A CGFSS is said to be a complex generalized absolute fuzzy soft set, denoted by $C \tilde{A}_{\alpha}(e)$, if $C \tilde{A}_{\alpha}(e): E \rightarrow I_{c}^{W} \times I_{c}$ such that $C \tilde{A}_{\alpha}(e)=(C A(e), \theta(e))$, where

$C A(e)=\overline{1} \cdot e^{i 2 \pi(1)} \forall e \in E$ and $\alpha(e)=1 . e^{i 2 \pi(1)} \forall e \in E$.

Theorem 3.13. Let $C F_{\mu}(e)$ be a CGFSS over $(W, E)$, then the following holds:

(i) $C F_{\mu}(e) \subseteq C F_{\mu}(e) \tilde{U} C F_{\mu}(e)$,

(ii) $C F_{\mu}(e) \tilde{\cap} C F_{\mu}(e) \subseteq C F_{\mu}(e)$,

(iii) $C F_{\mu}(e) \tilde{U} C \phi_{\theta}(e)=C F_{\mu}(e)$

(iv) $C F_{\mu}(e) \tilde{\cap} C \phi_{\theta}(e)=C \phi_{\theta}(e)$

(v) $C F_{\mu}(e) \tilde{U} C \tilde{A}_{\alpha}(e)=C \tilde{A}_{\alpha}(e)$

(vi) $C F_{\mu}(e) \tilde{U} C \tilde{A}_{\alpha}(e)=C F_{\mu}(e)$

Proof. Omitted.

Note 3.14. As a special case, we will have equality relation in (i) and (ii) above, if we take standard complex fuzzy operations (i.e. max, min, and standard complement).

Theorem 3.15. The following equalities are hold

$$
\begin{aligned}
& \text { (a) } C F_{\mu}(e) \tilde{U} C F_{\mu}{ }^{c}(e)=C \tilde{A}_{\alpha}(e) \text { and } \\
& \text { (b) } C F_{\mu} \tilde{U} C F_{\mu}{ }^{c}=C \phi_{\theta} .
\end{aligned}
$$

Proof. Omitted. 
Theorem 3.17. Let $C F_{\mu}(e), C G_{\delta}(e)$ and $C H_{\lambda}(e)$ be any three CGFSS over $(W, E)$, then:

(i) $C F_{\mu}(e) \cup \tilde{U} C G_{\delta}(e)=C G_{\delta}(e) \tilde{U} C F_{\mu}(e)$

(ii) $C F_{\mu}(e) \tilde{\cap} C G_{\delta}(e)=C G_{\delta}(e) \tilde{\cap} C F_{\mu}(e)$

(iii)

$$
C F_{\mu}(e) \tilde{U}\left(C G_{\delta}(e) \tilde{U} C H_{\lambda}(e)\right)=\left(C F_{\mu}(e) \tilde{U} C G_{\delta}(e)\right) \tilde{U} C H_{\lambda}(e)
$$

$$
C F_{\mu}(e) \tilde{\bigcap}\left(C G_{\delta}(e) \tilde{\cap} C H_{\lambda}(e)\right)=\left(C F_{\mu}(e) \tilde{\cap} C G_{\delta}(e)\right) \tilde{\cap} C H_{\lambda}(e) .
$$

.Proof. Omitted

Note 3.18. The distributive law does not hold on CGFSS in general, but as a special case for standard complex fuzzy operations then distributive law holds.

Theorem 3.19. Let $C F_{\mu}(e)$ and $C G_{\delta}(e)$ are two CGFSS over ( $W, E$ ), then the following holds:

$$
\left(C F_{\mu}(e) \tilde{\cap} C G_{\delta}(e)\right)^{c}=\left(C F_{\mu}{ }^{c}(e) \tilde{U} C G_{\delta}{ }^{c}(e)\right)
$$

(ii) $\left(C F_{\mu}(e) \tilde{U} C G_{\delta}(e)\right)^{c}=\left(C F_{\mu}{ }^{c}(e) \tilde{\cap} C G_{\delta}{ }^{c}(e)\right)$

Proof. Omitted.

\section{Complex Generalised Fuzzy Soft Relation}

To define a suitable application in decision-making problems to optimize the best solution we define CGFS relations and utilize it to find the best machine to be purchased from a seller $Z$.

Definition 4.1. Let $C F_{\mu}\left(s_{i}\right)$ and $C G_{\gamma}\left(s_{j}\right)$ be two CGFSS over $(W, E)$ and $Q \subseteq S^{2}$. then complex generalized fuzzy soft relation is a function $C R_{\lambda}\left(s_{i}, s_{j}\right): Q \rightarrow I_{c}{ }^{W} \times I_{c}$, defined as follows:

$C R_{\lambda}\left(s_{i}, s_{j}\right)=C F_{\mu}\left(s_{i}\right) \cap C G_{\gamma}\left(s_{j}\right)$ for all $\left(s_{i}, s_{j}\right) \in Q$, such that

$C R_{\lambda}\left(s_{i}, s_{j}\right)=\left(\operatorname{CR}\left(s_{i}, s_{j}\right), \lambda\left(s_{i}, s_{j}\right)\right)$,

where

$C R\left(s_{i}, s_{j}\right)=C F\left(s_{i}\right) \cap C G\left(s_{j}\right)=$

$\left\{\left(\left(s_{i}, s_{j}\right), \min \left(r_{C F\left(s_{i}\right)} \cap r_{C G\left(s_{j}\right)}\right) e^{i\left(\min \theta_{C F\left(s_{i}\right)}, \theta_{C G\left(s_{i}\right)}\right)}\right\}\right.$ and

$\lambda\left(s_{i}, s_{j}\right)=\mu\left(s_{i}\right) \cap \gamma\left(s_{j}\right)=$

$\left\{\left(\left(s_{i}, s_{j}\right), \min \left(r_{\mu\left(s_{i}\right)} \cap r_{\gamma\left(s_{j}\right)}\right) e^{i\left(\min \theta_{\mu\left(s_{i}\right)}, \theta_{\gamma\left(s_{i}\right)}\right)}\right\}^{\cdot}\right.$

An application in the decision-making problem and an illustration of the notion of complex generalized fuzzy soft relation are presented in application 4.1 bellow.

Application 4.1. Assume a firm $Y$ would like to purchase a machine from a seller $Z$. The seller $Z$ offers firm $Y$ three models of a machine with different production dates of each model. Consequently, a firm $Y$ has three models $\left(M_{1}, M_{2}\right.$, and $\left.M_{3}\right)$ to select with its production date $\left(D_{1}, D_{2}\right.$, and $D_{3}$ ) simultaneously. Let the company's team of analysts gives a possible value for three parameters that should be considered with the possibility for selecting the perfect time to buy machines (Purchase date). Parameters $(S)$ are: $s_{1}=$ durability, $s_{2}=$ purchasing cost, $S_{3}=$ maximum speed. These parameters will be influenced by some factors such as the purchase date (as an example: the price of the machine will cost a high price if it is manufactured in the present year (low price for machines manufactured in the past year). In other words, the team's opinion/decision depends on their experience and knowledge that may change depending on season or phase of purchasing a machine. As demonstrated above, CGFSS is the best way to represent this information (i.e., represent the data on people's choice that occurs periodically regarding possibility values of parameters that also may be changed periodically).

For more illustration, suppose that the firm's team has prepared an ideal machine with some possible parameters before getting the offer from seller $Z$. The goal of the team is to choose a proper machine recorded by seller $Z$ which is similar to the ideal machine. Let everyone in the selection team arrange in this stage for each machine's parameters a mark 1 or 0 to state whether the machine is proper for the parameter or not for each model and production date of the machine, and give 1 or 0 to state the possible value of parameters is proper or not for each parameter and purchase date of machines. For instance, suppose the $80 \%$ of the team of analysts trust that the ideal model machine is proper at the first parameter, and $60 \%$ of the team of analysts believe that is proper for the possible value of the first parameter, in which this manner is applied to compute the amplitude terms for membership functions in $\left(\mathrm{CF}_{\mu}(\mathrm{s})\right)$, in CGFS. The phase terms that present production date can be given for the first attribute of an ideal machine and the possibility of a purchasing date of the machine $60 \%$ and $50 \%$, respectively. The team trusts that the ideal production date of the machine is proper at the first attribute and possibility of purchasing date of the machine. So the ideal machine's first parameter can be presented as $0.8 e^{i 2 \pi(0.6)}$ and the possibility for the first parameter as $0.6 e^{i 2 \pi(0.5)}$. All information in this manner may be attained in the form of CGFSS as in the following tables, where both membership functions of CCFSS represents information of complex fuzzy set. 
Table 2. Ideal car model and production date with ideal possibility of parameters and possibility of purchase date.

\begin{tabular}{llll}
\hline Ideal $\mathrm{CF}_{\mu}(s)$ & $\mathbf{S 1}$ & $\mathbf{S 2}$ & $\mathrm{S3}$ \\
$\boldsymbol{M}_{\mathbf{1}}$ & $0.8 e^{i 2 \pi(0.6)}$ & $0.4 e^{i 2 \pi(0.3)}$ & $0.6 e^{i 2 \pi(0.4)}$ \\
$\boldsymbol{M}_{2}$ & $0.7 e^{i 2 \pi(0.8)}$ & $0.5 e^{i 2 \pi(0.7)}$ & $0.7 e^{i 2 \pi(0.7)}$ \\
$\boldsymbol{M x}_{3}$ & $0.4 e^{i 2 \pi(0.6)}$ & $0.8 e^{i 2 \pi(0.6)}$ & $0.6 e^{i 2 \pi(0.4)}$ \\
$\begin{array}{l}\text { Possibility } \\
\text { of } \mu \text { for }\end{array}$ & $0.6 e^{i 2 \pi(0.5)}$ & $0.6 e^{i 2 \pi(0.4)}$ & $0.4 e^{i 2 \pi(0.2)}$ \\
$\begin{array}{l}\text { parameter } \\
\text { and } \\
\text { possibility } \\
\text { perfect time } \\
\text { to purchase. }\end{array}$ & & & \\
\hline
\end{tabular}

So,

$$
\begin{aligned}
& C F_{\mu}\left(s_{1}\right)= \\
& \left(\left\{\frac{M_{1}}{0.8 e^{i 2 \pi(0.5)}}, \frac{M_{2}}{0.7 e^{i 2 \pi(0.8)}}, \frac{M_{3}}{0.4 e^{i 2 \pi(0.6)}}\right\}, 0.8 e^{i 2 \pi(0.5)}\right), \\
& C F_{\mu}\left(s_{2}\right)= \\
& \left(\left\{\frac{M_{1}}{0.4 e^{i 2 \pi(0.3)}}, \frac{M_{2}}{0.5 e^{i 2 \pi(0.7)}}, \frac{M_{3}}{0.8 e^{i 2 \pi(0.6)}}\right\}, 0.6 e^{i 2 \pi(0.4)}\right), \\
& C F_{\mu}\left(s_{3}\right)= \\
& \left(\left\{\frac{M_{1}}{0.6 e^{i 2 \pi(0.4)}}, \frac{M_{2}}{0.7 e^{i 2 \pi(0.7)}}, \frac{M_{3}}{0.6 e^{i 2 \pi(0.4)}}\right\}, 0.4 e^{i 2 \pi(0.2)}\right)
\end{aligned}
$$

Then $C F_{\mu}(s)$ is a CGFSS over $(W, E)$. and has the following matrix form,

$$
\begin{aligned}
& C F_{\mu}(x, \mu)= \\
& \left(\begin{array}{cccc}
0.8 e^{i 2 \pi(0.5)} & 0.7 e^{i 2 \pi(0.8)} & 0.4 e^{i 2 \pi(0.6)} & 0.8 e^{i 2 \pi(0.5)} \\
0.4 e^{i 2 \pi(0.3)} & 0.5 e^{i 2 \pi(0.7)} & 0.8 e^{i 2 \pi(0.6)} & 0.6 e^{i 2 \pi(0.4)} \\
0.6 e^{i 2 \pi(0.4)} & 0.7 e^{i 2 \pi(0.7)} & 0.6 e^{i 2 \pi(0.4)} & 0.4 e^{i 2 \pi(0.2)}
\end{array}\right) \\
& \\
&
\end{aligned}
$$

\begin{tabular}{llll}
\hline$M_{1}: C F_{\mu}(s)$ & $\boldsymbol{S 1}$ & $\boldsymbol{S} 2$ & $\boldsymbol{S 3}$ \\
$\boldsymbol{D}_{1}$ & $0.7 e^{i 2 \pi(0.7)}$ & $0.4 e^{i 2 \pi(0.6)}$ & $0.6 e^{i 2 \pi(0.5)}$ \\
$\boldsymbol{D}_{2}$ & $0.6 e^{i 2 \pi(0.4)}$ & $0.5 e^{i 2 \pi(0.8)}$ & $0.5 e^{i 2 \pi(0.8)}$ \\
$\boldsymbol{D}_{3}$ & $0.9 e^{i 2 \pi(1)}$ & $0.8 e^{i 2 \pi(0.6)}$ & $0.5 e^{i 2 \pi(0.6)}$
\end{tabular}

Possibility of $\mu$ for parameter $\quad 0.5 e^{i 2 \pi(0.6)} \quad 0.7 e^{i 2 \pi(0.6)} \quad 0.6 e^{i 2 \pi(0.7)}$ and
And has the following matrix form,

$$
\begin{aligned}
& M_{1}: C F_{\mu}(x, \mu)= \\
& \left(\begin{array}{llll}
0.7 e^{i 2 \pi(0.7)} & 0.6 e^{i 2 \pi(0.4)} & 0.9 e^{i 2 \pi(1)} & 0.5 e^{i 2 \pi(0.6)} \\
0.4 e^{i 2 \pi(0.6)} & 0.5 e^{i 2 \pi(0.8)} & 0.8 e^{i 2 \pi(0.6)} & 0.7 e^{i 2 \pi(0.6)} \\
0.6 e^{i 2 \pi(0.5)} & 0.5 e^{i 2 \pi(0.8)} & 0.5 e^{i 2 \pi(0.6)} & 0.6 e^{i 2 \pi(0.7)}
\end{array}\right)
\end{aligned}
$$

Similarly we compute the following matrix form of $M_{2}: C F_{\mu}(x, \mu)$ and $M_{3}: C F_{\mu}(x, \mu)$,

$$
\begin{aligned}
& M_{2}: C F_{\mu}(x, \mu)= \\
& \left(\begin{array}{llll}
0.6 e^{i 2 \pi(0.5)} & 0.7 e^{i 2 \pi(0.6)} & 0.8 e^{i 2 \pi(0.8)} & 0.7 e^{i 2 \pi(0.6)} \\
0.7 e^{i 2 \pi(0.4)} & 0.8 e^{i 2 \pi(0.5)} & 0.7 e^{i 2 \pi(0.8)} & 0.5 e^{i 2 \pi(0.4)} \\
0.4 e^{i 2 \pi(0.7)} & 0.4 e^{i 2 \pi(0.8)} & 0.0 e^{i 2 \pi(0.0)} & 0.8 e^{i 2 \pi(0.6)}
\end{array}\right)
\end{aligned}
$$

And

$$
\begin{aligned}
& M_{3}: \mathrm{CF}_{\mu}(x, \mu)= \\
& \left(\begin{array}{cccc}
0.7 e^{i 2 \pi(0.8)} & 0.9 e^{i 2 \pi(0.3)} & 0.8 e^{i 2 \pi(0.5)} & 0.8 e^{i 2 \pi(0.7)} \\
0.8 e^{i 2 \pi(0.3)} & 0.5 e^{i 2 \pi(0.5)} & 0.4 e^{i 2 \pi(0.3)} & 0.4 e^{i 2 \pi(0.7)} \\
0.4 e^{i 2 \pi(0.5)} & 0.9 e^{i 2 \pi(0.6)} & 0.8 e^{i 2 \pi(0.2)} & 0.6 e^{i 2 \pi(0.5)}
\end{array}\right)
\end{aligned}
$$

To keep going in our application, we use a suitable model to choose a proper machine that is most similar to the ideal machine (see [9]). And generalized it to deal with complex fuzzy information as follows.

Model: In this model, we are using the Normalized Euclidean distance between two complex fuzzy sets [14]

$$
q_{C F}(A, B)=\sqrt{\frac{1}{2 n} \sum_{i=1}^{n}\left[\left(r_{A}\left(x_{i}\right)-r_{B}\left(x_{i}\right)\right)^{2}+\frac{1}{2 \pi^{2}}\left(\theta_{A}\left(x_{i}\right)-\theta_{B}\left(x_{i}\right)\right)^{2}\right]}
$$

1) Evaluate the distance between $M_{1}$ in Eq. 1 and Eq. 2, $M_{2}$ in Eq. 1 and Eq. 3, and $M_{3}$ in Eq. 1 and Eq. 4, to find the most similar production date to the ideal machine (We seek to get the machine with production date that has a minimum value of distance compared to the ideal machine).

$$
\begin{aligned}
q_{C F}\left(M_{1}, D_{1}\right) & =\sqrt{\frac{1}{6} \sum_{i=1}^{3}\left[\frac{\left[(0.8-0.7)^{2}+(0.4-0.4)^{2}+(0.6-0.6)^{2}\right]+}{2 \pi^{2}}\left[4 \pi^{2}(0.6-0.7)^{2}+4 \pi^{2}(0.3-0.6)^{2}+4 \pi^{2}(0.4-0.5)^{2}\right]\right]} \\
= & \sqrt{\frac{1}{6} \sum_{i=1}^{3}[[0.01]+2[0.11]]}=0.195789002
\end{aligned}
$$

Similarly, we evaluate $q_{C F}\left(M_{1}, D_{2}\right)=0.4$,

$$
\begin{aligned}
& q_{C F}\left(M_{1}, D_{3}\right)=0.3559026084, \\
& q_{C F}\left(M_{2}, D_{1}\right)=0.2886751345, \\
& q_{C F}\left(M_{2}, D_{2}\right)=0.2449489743, \\
& q_{C F}\left(M_{2}, D_{3}\right)=0.5066228051, \\
& q_{C F}\left(M_{3}, D_{1}\right)=0.2614064524,
\end{aligned}
$$

and 
$q_{C F}\left(M_{3}, D_{2}\right)=0.343996124$,

$q_{C F}\left(M_{3}, D_{3}\right)=0.3265986324$.

2) Evaluate the distance between possibility parameters $s_{i}, i=1,2$, and 3 , in table 1 and possibility parameters in each table $j, j=1,2$, and 3 separately. To find the most similar possibility parameter and its purchase date to the ideal machine. (We seek to get the possibility parameters with purchase date that has a minimum value of distance compared to the ideal possibility parameters and its purchase date).

$$
\begin{aligned}
& q_{C F}\left(s_{1, M_{1}}, s_{1}\right)=\sqrt{\frac{1}{2} \sum_{i=1}^{1}\left[\left[(0.5-0.6)^{2}\right]+\frac{1}{2 \pi^{2}}\left[4 \pi^{2}(0.6-0.5)^{2}\right]\right]} \\
& =\sqrt{\frac{1}{2} \sum_{i=1}^{1}[0.01+2[0.01]]}=0.1224744871
\end{aligned}
$$

Similarly, we evaluate

$$
\begin{aligned}
& q_{C F}\left(s_{1, M_{2}}, s_{1}\right)=0.1224744871, \\
& q_{C F}\left(s_{1, M_{3}}, s_{1}\right)=0.2449489743, \\
& q_{C F}\left(s_{2, M_{1}}, s_{2}\right)=0.2121320344, \\
& q_{C F}\left(s_{2, M_{2}}, s_{2}\right)=0.0707106781, \\
& q_{C F}\left(s_{2, M_{3}}, s_{2}\right)=0.331662479, \\
& q_{C F}\left(s_{3, M_{1}}, s_{3}\right)=0.5744562647, \\
& q_{C F}\left(s_{3, M_{2}}, s_{3}\right)=0.4898979486, \text { and } \\
& q_{C F}\left(s_{3, M_{3}}, s_{3}\right)=0.331662479 .
\end{aligned}
$$

3) After identifying the desirable production date for each machine and the most possibility parameters to the similar ideal machine and its possibility parameters respectively in steps one and two. We generalize a new CGFSS containing the three machines and parameters identified in steps one and two.

Thus, the minimum value between the ideal machine and best production date is 0.195789002 which implies the best production date for the first machine is $D_{1}$. Also, the minimum value between

\begin{tabular}{|c|c|c|c|}
\hline \multicolumn{4}{|l|}{ Calculated } \\
\hline$C G_{\mu}(s)$ & $S 1$ & $S 2$ & S3 \\
\hline$M_{1}$ & $0.7 e^{i 2 \pi(0.7)}$ & $0.4 e^{i 2 \pi(0.6)}$ & $0.6 e^{i 2 \pi(0.5)}$ \\
\hline$M_{2}$ & $0.7 e^{i 2 \pi(0.6)}$ & $0.8 e^{i 2 \pi(0.5)}$ & $0.4 e^{i 2 \pi(0.8)}$ \\
\hline$M_{3}$ & $0.7 e^{i 2 \pi(0.8)}$ & $0.8 e^{i 2 \pi(0.3)}$ & $0.4 e^{i 2 \pi(0.5)}$ \\
\hline $\begin{array}{l}\text { Possibility } \\
\text { of } \mu \text { for } \\
\text { parameter } \\
\text { and } \\
\text { possibility }\end{array}$ & $0.5 e^{i 2 \pi(0.6)}$ & $0.5 e^{i 2 \pi(0.4)}$ & $0.6 e^{i 2 \pi(0.5)}$ \\
\hline
\end{tabular}
the ideal possibility machine parameters and its purchase date comparing with parameters $s_{1}, s_{2}$, and $s_{3}$, for each machine, are " 0.1224744871 ", “ 0.0707106781 ", and " 0.331662479 ", which implies the best possible parameters for $s_{1}, s_{2}$, and $s_{3}$, respectively. Similarly for the second and third machines. The result of calculated CGFSS “ $C G_{\mu}(s)$ " is presented in Table 4.
Table 4. Calculated $C G_{\mu}(s)$

4) Define the relation between two CGCFSS: Ideal $C_{\mu}(s)$ and Calculated $C G_{\mu}(s)$.

$$
\left(\begin{array}{lcccc}
R & M_{1} & M_{2} & M_{3} & \lambda \\
\left(s_{1}, s_{1}\right) & 0.7 e^{i 2 \pi(0.6)} & 0.7 e^{i 2 \pi(0.6)} & 0.4 e^{i 2 \pi(0.6)} & 0.5 e^{i 2 \pi(0.5)} \\
\left(s_{1}, s_{2}\right) & 0.4 e^{i 2 \pi(0.6)} & 0.7 e^{i 2 \pi(0.6)} & 0.4 e^{i 2 \pi(0.6)} & 0.5 e^{i 2 \pi(0.4)} \\
\left(s_{1}, s_{3}\right) & 0.6 e^{i 2 \pi(0.5)} & 0.4 e^{i 2 \pi(0.8)} & 0.4 e^{i 2 \pi(0.5)} & 0.6 e^{i 2 \pi(0.5)} \\
\left(s_{2}, s_{1}\right) & 0.4 e^{i 2 \pi(0.3)} & 0.5 e^{i 2 \pi(0.6)} & 0.7 e^{i 2 \pi(0.6)} & 0.5 e^{i 2 \pi(0.4)} \\
\left(s_{2}, s_{2}\right) & 0.4 e^{i 2 \pi(0.3)} & 0.5 e^{i 2 \pi(0.5)} & 0.8 e^{i 2 \pi(0.3)} & 0.5 e^{i 2 \pi(0.4)} \\
\left(s_{2}, s_{3}\right) & 0.4 e^{i 2 \pi(0.3)} & 0.4 e^{i 2 \pi(0.7)} & 0.4 e^{i 2 \pi(0.5)} & 0.6 e^{i 2 \pi(0.4)} \\
\left(s_{3}, s_{1}\right) & 0.6 e^{i 2 \pi(0.4)} & 0.6 e^{i 2 \pi(0.4)} & 0.6 e^{i 2 \pi(0.4)} & 0.4 e^{i 2 \pi(0.2)} \\
\left(s_{3}, s_{2}\right) & 0.4 e^{i 2 \pi(0.4)} & 0.7 e^{i 2 \pi(0.5)} & 0.6 e^{i 2 \pi(0.3)} & 0.4 e^{i 2 \pi(0.2)} \\
\left(s_{3}, s_{3}\right) & 0.6 e^{i 2 \pi(0.4)} & 0.4 e^{i 2 \pi(0.7)} & 0.4 e^{i 2 \pi(0.4)} & 0.4 e^{i 2 \pi(0.2)}
\end{array}\right)
$$

5) In this step, we generalize the model in [9] to deal with complex fuzzy information. Let us get started to accomplish the best machine by adding the amplitude term and phase term values for each value in the relation table above. Then mark the maximum numerical grade (indicated in parenthesis) in each row excluding the last column which is the grade of such belongingness of a machine against each pair of parameters. Now the score of each of such machines is calculated by taking the sum of the products of these numerical grades with the corresponding values of $\lambda$. The machine with the maximum score is the wanted machine. We do not consider the numerical grades of the machines against the pairs $\left(e_{i}, e_{i}\right), i=1,2,3$, as both the parameters are the same. 


$$
\left(\begin{array}{lllcl}
R & M_{1} & M_{2} & M_{3} & \lambda \\
\left(s_{1}, s_{1}\right) & (1.3) & (1.3) & 1 & 1 \\
\left(s_{1}, s_{2}\right) & 1 & (1.3) & 1 & 0.9 \\
\left(s_{1}, s_{3}\right) & 1.1 & (1.2) & 0.9 & 1.1 \\
\left(s_{2}, s_{1}\right) & 0.7 & 1.1 & (1.3) & 0.9 \\
\left(s_{2}, s_{2}\right) & 0.7 & 1 & (1.1) & 0.9 \\
\left(s_{2}, s_{3}\right) & 0.7 & (1.1) & 0.9 & 1 \\
\left(s_{3}, s_{1}\right) & (1) & (1) & (1) & 0.6 \\
\left(s_{3}, s_{2}\right) & 0.8 & (1.2) & 0.9 & 0.6 \\
\left(s_{3}, s_{3}\right) & 1 & (1.1) & 0.8 & 0.6
\end{array}\right)
$$

$\left(\begin{array}{cccccccccc}\multicolumn{8}{c}{\text { Grade table }} \\ \text { CR } & \left(s_{1}, s_{1}\right) & \left(s_{1}, s_{2}\right) & \left(s_{1}, s_{3}\right) & \left(s_{2}, s_{1}\right) & \left(s_{2}, s_{2}\right) & \left(s_{2}, s_{3}\right) & \left(s_{3}, s_{1}\right) & \left(s_{3}, s_{2}\right) & \left(s_{3}, s_{3}\right) \\ M & M_{1}, M_{2} & M_{2} & M_{2} & M_{3} & M_{3} & M_{2} & M_{1}, M_{2}, M_{3} & M_{2} & M_{2} \\ \begin{array}{c}\text { Highest numerical } \\ \text { grade }\end{array} & 1.3 & 1.3 & 1.2 & 1.3 & 1.1 & 1.1 & 1 & 1.2 & 1.1 \\ \lambda & & & & & & & & & \\ \lambda & & 0.9 & 1.1 & 0.9 & & 1 & 0.6 & 0.6 & \end{array}\right)$

Score $\left(M_{1}\right)=1 \times 0.6=0.6 \quad, \quad$ score $\quad\left(M_{2}\right)=$ $1.3 \times 0.9+1.2 \times 1.1+1.1 \times 1+1 \times 0.6+1.2 \times 0.6=4.91$ , and score $\left(M_{3}\right)=1.3 \times 0.9+1 \times 0.6=1.77$

Then the firm's team will choose the machine with maximum score. Therefore the team will buy machine $M_{2}$.

\section{Different uncertainty sets with CGFSS}

We extend the range of belongingness and possibility of belongingness terms of GFSS as presented by Majumdar and Samanta [9] to convey CGFSS information. The new concept of CGFSS can be employed to simultaneously represent the uncertainty and periodicity problems of GFSS information in complex geometry. We used a polar form $\left(r \cdot e^{i \theta}\right)$ to show the advantage of the nature of periodic that appears in the complex-valued membership functions. See Table 5. In next page.

\section{Conclusion}

We introduced complex generalized fuzzy soft set by employing GFSS to the phase term in the complex numbers. The advantage of the CGFSS may be concluded by its ability to represent problems. Not only the parametric features of the problems, but also the features are affected by a factor that also conveys GFSS information simultaneously. CGFSS can be useful to represent the information several problems in decisionmaking by using two variables instead of one variable. A comparative study among different uncertainty sets with CGFSS has illustrated. The limitation of CGFS may appeared in indicating a membership and non-membership values for a collection of approximate descriptions of an object. This limitation can be enclosed in future researches by combining CGFSS and the innovative concept called intuitionistic fuzzy set [29]. CGFSS has no ability to characterize the truth, falsity, and intermediate information as in complex neutrosophic set, So the future concept may be introduced is complex neutrosophic generalized fuzzy soft set [30]. 
Table 5. Comparative study among different uncertainty sets (FS, FSS, GFSS, CFS) with CGFSS

\begin{tabular}{|c|c|c|c|c|c|}
\hline & FS & FSS & GFSS & CFS & CGFS \\
\hline Domain & $\begin{array}{l}\text { Universe of } \\
\text { discourse }\end{array}$ & $\begin{array}{c}\text { Universe of } \\
\text { discourse }\end{array}$ & $\begin{array}{c}\text { Universe of } \\
\text { discourse }\end{array}$ & $\begin{array}{l}\text { Universe of } \\
\text { discourse }\end{array}$ & $\begin{array}{c}\text { Universe of } \\
\text { discourse }\end{array}$ \\
\hline Co-domain & $\begin{array}{c}\text { Single- } \\
\text { valued in } \\
{[0,1]}\end{array}$ & $\begin{array}{c}\text { Single- } \\
\text { valued in } \\
{[0,1]}\end{array}$ & $\begin{array}{c}\text { Two values in } \\
{[0,1]}\end{array}$ & $\begin{array}{c}\text { One value in } \\
{[0,1] e^{i[0,1]}}\end{array}$ & $\begin{array}{l}\text { Two Values in } \\
{[0,1] e^{[0,1]}}\end{array}$ \\
\hline degree of belongingness & Yes & Yes & Yes & Yes & Yes \\
\hline $\begin{array}{l}\text { degree of possibility of } \\
\text { belongingness }\end{array}$ & No & No & Yes & No & Yes \\
\hline Uncertainty measurement & Yes & Yes & Yes & Yes & Yes \\
\hline Periodic measurement & No & No & No & Yes & Yes \\
\hline Unit disk & No & No & No & Yes & Yes \\
\hline $\begin{array}{l}\text { Amplitude term (degree } \\
\text { of belongingness) }\end{array}$ & $\begin{array}{c}\text { Single } \\
\text { valued in } \\
{[0,1]}\end{array}$ & $\begin{array}{c}\text { Single } \\
\text { valued in } \\
{[0,1]}\end{array}$ & $\begin{array}{l}\text { Single valued } \\
\text { in }[0,1]\end{array}$ & $\begin{array}{c}\text { Single } \\
\text { valued in } \\
{[0,1]}\end{array}$ & $\begin{array}{l}\text { Single valued in } \\
{[0,1]}\end{array}$ \\
\hline $\begin{array}{l}\text { Amplitude term (degree } \\
\text { of possibility of } \\
\text { belongingness) }\end{array}$ & No & No & $\begin{array}{l}\text { Single valued } \\
\text { in }[0,1]\end{array}$ & No & $\begin{array}{c}\text { Single valued in } \\
{[0,1]}\end{array}$ \\
\hline $\begin{array}{c}\text { phase term } \\
\text { (degree of belongingness) }\end{array}$ & No & No & No & $\begin{array}{c}\text { Single } \\
\text { valued in } \\
{[0,1]}\end{array}$ & $\begin{array}{c}\text { Single valued in } \\
{[0,1]}\end{array}$ \\
\hline $\begin{array}{c}\text { phase term } \\
\text { (degree of possibility of } \\
\text { belongingness) }\end{array}$ & No & No & No & No & $\begin{array}{l}\text { Single valued in } \\
{[0,1]}\end{array}$ \\
\hline $\begin{array}{l}\text { Ability to convey GFSS } \\
\text { (Parametrization nature } \\
\text { and its possibility) }\end{array}$ & No & No & $\begin{array}{l}\text { Yes, only for } \\
\text { the amplitude } \\
\text { term }\end{array}$ & No & $\begin{array}{l}\text { Yes, for both } \\
\text { amplitude and } \\
\text { phase terms. }\end{array}$ \\
\hline
\end{tabular}

\section{References}

1. Tran Thi Ngan, Luong Thi Hong Lan, Mumtaz Ali, Dan Tamir, Le Hoang SON, Tran Manh Tuan, Naphtali RISHE and Abe Kandel. Logic Connectives of Complex Fuzzy Sets. Romanian Journal of Information Science and Technology. 21 (4). 2018. 344-357.

2. Li C., \& Chiang T. W. Complex Neurofuzzy ARIMA Forecasting - A New Approach Using Complex Fuzzy Sets. IEEE Transactions on Fuzzy Systems. 21(3), 2013.567-584.

3. Yousef Al-Qudah, Mazlan Hassan, and Nasruddin Hassan. Fuzzy Parameterized Complex Multi-Fuzzy Soft Expert Set Theory and Its Application in Decision-Making. Symmetry. $\quad 11, \quad 358 . \quad 2019$.; doi:10.3390/sym11030358.

4. P. K. Maji, A. R. Roy, and R. Biswas. Fuzzy Soft Sets. Journal of Fuzzy Mathematics, 9, 2001.589-602.
5. P. Kmaji, Etal. An application of soft sets in a decision-making problem. Comput. Math. Appl. 44. 2002.1077-1083.

6. P. Kmaji, Etal. Soft set theory. Comput. Math. Appl.45. 2003. 555-562.

7. D. Molodtsov. Soft set theory-First results. Comput. Math. Appl. 37. 1999. 19-31.

8. Z. Kong, Etal. Comment on A Fuzzy Soft SetTheoretic Approach to Decision Making Problems. J. Comput. Appl. Math. 223. 2009. 540-542.

9. P. Majumdar, and S.K. Samanta. Generalised Fuzzy Soft Sets. Computers and Mathematics with Applications. 59. 2010. 1425-1432.

10. Alkouri, A., \& Salleh, A. Complex Atanassov's Intuitionistic Fuzzy Relation. Journal of Abstract and Applied Analysis, Article ID 287382. 2013.218 pages, doi:10.1155/2013/287382.

11. Alkouri, A. \& Salleh, A. Complex Atanassov's intuitionistic fuzzy Set. in AIP Conf. Proc., 
International conference on fundamental and applied sciences, Kuala Lampur, Malaysia. 2012. 464-470; doi: 10. 1063/1.4757515.

12. Alkouri And Salleh. Some operations on complex Atanassov's intuitionistic fuzzy sets. AIP conference proceedings. 1571, (1): 2012. 987-993.

13. Alkouri And Salleh. Complex fuzzy soft multisets. AIP Conference Proceedings. 1614, (1): 2012. 955-961.

14. Alkouri And Salleh. Linguistic variable, hedges and several distances on complex fuzzy sets. Journal of Intelligent \& Fuzzy Systems. 26. 2014. 2527-2535 DOI:10.3233/IFS-130923 IOS Press.

15. Lee, K. H. First Course on Fuzzy Theory and Applications. Springer-Verlag, 2004.

16. Li, C. \& Chiang, T.-W. Complex Fuzzy Computing to Time Series Prediction-A MultiSwarm Pso Learning Approach," ACIIDS, Lecture Notes In Artificial Intelligence. 6592:. 2011. 242-251.

17. Li, C. \& Chiang, T.-W. Complex Fuzzy Model with PSO-RLSE Hybrid Learning Approach to Function Approximation. International Journal of Intelligent Information and Database Systems. 5, (4). 2011. 409-430.

18. Li, C. \& Chiang, T.-W. Complex Neurofuzzy ARIMA Forecasting A New Approach Using Complex Fuzzy Sets," IEEE Transactions On Fuzzy Systems, 21, (3). 2013. 567-584.

19. Li, C. \& Chiang, T.-W. Complex Neuro-Fuzzy Self-Learning Approach to Function Approximation. Lecture Notes in Artificial Intelligence. 5991. 2010. 289-299.

20. Li, C., Wu, T. \& Chan, F.-T. Self-Learning Complex Neuro-Fuzzy System with Complex Fuzzy Sets and Its Application to Adaptive Image Noise Canceling. Neurocomputing. 94, (1). 2012. 121-139.

21. Ma, J., Zhang G. \& Lu, J. A Method for Multiple Periodic Factor Prediction Problems Using Complex Fuzzy Sets. IEEE Trans. On Fuzzy. System. 20, (1). 2012. 32-45.

22. Ganeshsree Selvachandran, Nisren A. Hafeed, and Abdul Razak Salleh. Complex Fuzzy Soft Expert Sets. AIP Conference Proceedings 1830, 070020. 2017. Doi: 10.1063/1.4980969.

23. Ma, J., Zhang G. \& Lu, J. A Method for Multiple Periodic Factor Prediction Problems Using Complex Fuzzy Sets. IEEE Trans. on Fuzzy. System. 20, (1). 2012. 32-45.

24. Ramot, D., Milo, R., Friedman, M., \& A. Kandel, A. Complex Fuzzy Sets. IEEE
Transaction on Fuzzy Systems10. 2002. 171186.

25. Yousef Al-Qudah and Nasruddin Hassan. Complex Multi-Fuzzy Relation for Decision Making Using Uncertain Periodic Data. International Journal of Engineering \& Technology. 7 (4). 2018. 2437-2445.

26. Zadeh, A. Fuzzy Sets". Inform. Control. 8. 1965. 338-353.

27. Zhang, G., Dillon, T. S., Cai, K. Y., Ma, J. \& Lu, J. Operation Properties and Delta-Equalities of Complex Fuzzy Sets," International Journal of Approximate Reasoning, 50, (8). 2009. 12271249.

28. Ramot, D., Milo, R., Friedman, M. \& Kandel, A. Complex Fuzzy Sets. IEEE Transactions on Fuzzy Systems. 10, (2). 2002. 171-186.

29. K. Atanassov, Intuitionistic Fuzzy Sets, PhysicaVerlag, Heidelberg. 1999.

30. M. Ali and F. Smarandache, Complex neutrosophic set. Neural Computing and Applications, 2017, 28. (7). 2017. 1817-1834.

\section{Creative Commons Attribution License 4.0 (Attribution 4.0 International , CC BY 4.0)}

This article is published under the terms of the Creative Commons Attribution License 4.0 https://creativecommons.org/licenses/by/4.0/deed.en_US 